\title{
INTEGRABILITY OF DOUBLE LACUNARY SINE SERIES
}

\author{
FERENC MÓRICZ
}

(Communicated by J. Marshall Ash)

\begin{abstract}
We consider mainly the series $\sum \sum a_{j k} \sin 2^{j} x \sin 2^{k} y$, which converges to a finite function $f(x, y)$ a.e. if $\sum \sum a_{j k}^{2}<\infty$. We show that the (Lebesgue) integrability of $x^{-1} y^{-1} f(x, y)$ over the two-dimensional torus is essentially controlled by the quantity $\sum_{m=1}^{\infty} \sum_{n=1}^{\infty}\left(\sum_{j=m}^{\infty} \sum_{k=n}^{\infty} a_{j k}^{2}\right)^{1 / 2}$. Our result is an extension of the corresponding one by $M$. C. Weiss [4] from onedimensional to two-dimensional lacunary sine series.
\end{abstract}

\section{INTRODUCTION}

First we consider the double sine series

$$
\sum_{j=1}^{\infty} \sum_{k=1}^{\infty} a_{j k} \sin m_{j} x \sin n_{k} y
$$

on the positive quadrant $T_{+}^{2}=[0, \pi] \times[0, \pi]$ of the two-dimensional torus, where the $a_{j k}$ are real numbers and the $m_{j}, n_{k}$ are positive integers satisfying the Hadamard lacunarity condition in both variables:

$$
\frac{m_{j+1}}{m_{j}} \geq q>1 \text { and } \frac{n_{k+1}}{n_{k}} \geq q>1 \quad(j, k=1,2 \ldots) .
$$

As in the one-dimensional case (see, e.g., [5, Vol. 1, p. 79]), if

$$
\sum_{j=1}^{\infty} \sum_{k=1}^{\infty} a_{j k}^{2}<\infty
$$

then series (1.1) converges a.e. For the reader's convenience, we sketch a proof

Received by the editors March 6, 1989 and, in revised form, July 31, 1989.

1980 Mathematics Subject Classification (1985 Revision). Primary 42B05; Secondary 42A55.

Key words and phrases. Double sine series, Fourier series, lacunarity, a.e. convergence, Lebesgue integrability. 
here. Denote by $s_{m n}(x, y)$ and $\sigma_{m n}(x, y)$ the rectangular partial sums and the arithmetic means of series (1.1) with the vacant terms replaced by zeros. It is well known (see, e.g. [5, Vol. 2, p. 308]) that the double sequence $\sigma_{m n}(x, y)$ converges a.e. to the finite function $f(x, y)$, which corresponds to series (1.1) by the two-dimensional Riesz-Fischer theorem, owing to (1.3). On the other hand, by lacunarity,

$$
\begin{aligned}
\left(m_{j+1}-m_{j}\right)\left(n_{k+1}-n_{k}\right) s_{m_{j}, n_{k}}= & m_{j+1} n_{k+1} \sigma_{m_{j+1}-1, n_{k+1}-1}-m_{j} n_{k+1} \sigma_{m_{j}-1, n_{k+1}-1} \\
& -m_{j+1} n_{k} \sigma_{m_{j+1}-1, n_{k}-1}+m_{j} n_{k} \sigma_{m_{j}-1, n_{k}-1}
\end{aligned}
$$

(we omitted $x, y$ for brevity). Hence, given any $\varepsilon>0$,

$$
\left(1-\frac{m_{j}}{m_{j+1}}\right)\left(1-\frac{n_{k}}{n_{k+1}}\right)\left|s_{m_{j}, n_{k}}(x, y)-f(x, y)\right| \leq 4 \varepsilon
$$

and, by (1.2),

$$
\left|s_{m_{j}, n_{k}}(x, y)-f(x, y)\right| \leq \frac{4 q^{2} \varepsilon}{(q-1)^{2}}
$$

if $j$ and $k$ are large enough, provided $\sigma_{m n}(x, y)$ converges to $f(x, y)$. By what we have said above, it follows that $s_{m_{j}, n_{k}}(x, y)$ converges to $f(x, y)$ a.e.

It remains only to observe that in our lacunary case this is equivalent to the a.e. convergence of $s_{m n}(x, y)$.

Remark 1. Without proof we note that a converse statement is also true. More precisely, if series (1.1) converges on a set $E$ of points $(x, y)$ of positive planar measure, then (1.3) is satisfied. This can be verified by applying a generalized version of Lemma 1 in $\S 4$ below, when the left-hand side of (4.1) is replaced by

$$
\iint_{E}\left|\sum_{j=1}^{\infty} \sum_{k=1}^{\infty} a_{j k} \sin m_{j} x \sin n_{k} y\right| d x d y .
$$

Remark 2. The whole $\S 1$ applies to the double lacunary cosine series

$$
\sum_{j=1}^{\infty} \sum_{k=1}^{\infty} a_{j k} \cos m_{j} x \cos n_{k} y
$$

and mixed series

$$
\sum_{j=1}^{\infty} \sum_{k=1}^{\infty} a_{j k} \cos m_{j} x \sin n_{k} y
$$

The methods described below clearly apply to multiple lacunary trigonometric series, and the extension of the results to the $d$-multiple case, where $d \geq 3$ is an integer, is straightforward. However, we consider only double series in order to keep the notation manageable. 


\section{MAIN RESULtS}

Our goal is to study the integrability of $x^{-1} y^{-1} f(x, y)$ over $T_{+}^{2}$ in the particular case when (1.1) is of the form

$$
\sum_{j=1}^{\infty} \sum_{k=1}^{\infty} a_{j k} \sin 2^{j} x \sin 2^{k} y=: f(x, y),
$$

which exists a.e. in the case of (1.3).

Theorem 1. Assume condition (1.3) is satisfied. If

$$
\sum_{m=1}^{\infty} \sum_{n=1}^{\infty}\left(\sum_{j=m}^{\infty} \sum_{k=n}^{\infty} a_{j k}^{2}\right)^{1 / 2}<\infty
$$

then

$$
x^{-1} y^{-1} f(x, y) \in L\left(T_{+}^{2}\right) .
$$

Remark 3. Condition (2.2) is equivalent to the simultaneous fulfillment of the following three conditions:

$$
\begin{aligned}
& \sum_{m=1}^{\infty} \sum_{n=1}^{\infty}\left(\sum_{j=m+1}^{\infty} \sum_{k=n+1}^{\infty} a_{j k}^{2}\right)^{1 / 2}<\infty \\
& \sum_{j=1}^{\infty}\left|a_{j 1}\right|<\infty \text { and } \sum_{k=1}^{\infty}\left|a_{1 k}\right|<\infty
\end{aligned}
$$

In order to prove that condition (2.2) is also necessary for (2.3) we need the following assumptions:

$$
\sum_{m=1}^{\infty}\left(\sum_{j=m}^{\infty} a_{j k}^{2}\right)^{1 / 2}<\infty \quad(k=1,2 \ldots)
$$

and

$$
\sum_{n=1}^{\infty}\left(\sum_{k=n}^{\infty} a_{j k}^{2}\right)^{1 / 2}<\infty \quad(j=1,2 \ldots) .
$$

These conditions are weaker than (2.2) and a forthcoming explanation makes them quite natural.

Theorem 2. Assume conditions (1.3), (2.4), and (2.5) are satisfied. Then (2.3) implies (2.2).

We observe that condition (2.2) actually implies more than (2.3). To explain this, consider the so-called "row" series (i.e. when $k$ is fixed and $\sin 2^{k} y$ is 
deleted in series (2.1)):

$$
\sum_{j=1}^{\infty} a_{j k} \sin 2^{j} x=: g_{k}(x) \quad(k=1,2 \ldots)
$$

and the "column" series

$$
\sum_{k=1}^{\infty} a_{j k} \sin 2^{k} y=: h_{j}(y) \quad(j=1,2 \ldots) .
$$

The a.e. convergence of these series follows also from (1.3). According to the corresponding one-dimensional theorem of M. C. Weiss [4],

$$
x^{-1} g_{k}(x) \in L\left(T_{+}\right) \quad(k=1,2 \ldots)
$$

if and only if condition (2.4) is satisfied; and

$$
y^{-1} h_{j}(y) \in L\left(T_{+}\right) \quad(j=1,2 \ldots)
$$

if and only if condition (2.5) is satisfied; here $T_{+}=[0, \pi]$.

Now it is plain that condition (2.2) implies not only the fulfillment of (2.3) (by virtue of Theorem 1), but also that of (2.6) and (2.7) (by virtue of the theorem of M. C. Weiss cited above). And this time the converse is also true. In fact, if conditions (2.6) and (2.7) are satisfied, then we conclude (2.4) and (2.5), and in turn, we can apply Theorem 2 to deduce (2.2) from (2.3). Thus, we have the following characterization.

Corollary. Assume that condition (1.3) is satisfied, $f(x, y)$ is the sum of series (2.1), the $g_{k}(x)$ are the sums of the row series, and the $h_{j}(y)$ are the sums of the column series of (2.1). Then (2.2) is a necessary and sufficient condition for the simultaneous fulfillment of the integrability conditions (2.3), (2.6), and (2.7).

These results can be considered as the extension of the theorem of M. C. Weiss [4] from one-dimensional to two-dimensional lacunary sine series. The proofs also make use of some ideas occurring in [1, pp. 32-33].

Remark 4. Keeping in mind the more general result of [4] (cf. [1, Theorem 5.27]) we formulate the following

Conjecture. Assume that conditions (1.2) and (1.3) are satisfied, $f(x, y)$ is the sum of series (1.1), the $g_{k}(x)$ are the sums of the row series, and the $h_{j}(y)$ are the sums of the column series of (1.1). Then the integrability conditions (2.3), (2.6), and (2.7) are satisfied if and only if

$$
\sum_{j=1}^{\infty} \sum_{k=1}^{\infty}\left(\ln \frac{m_{j+1}}{m_{j}}\right)\left(\ln \frac{n_{k+1}}{n_{k}}\right)\left(\sum_{i=j}^{\infty} \sum_{l=k}^{\infty} a_{i l}^{2}\right)^{1 / 2}<\infty .
$$




\section{Proof of Theorem 1}

Let $M$ and $N$ be positive integers. Then we can estimate as follows

$$
\begin{aligned}
& I_{M N}:=\int_{\pi 2^{-M-1}}^{\pi} \int_{\pi 2^{-N-1}}^{\pi} \frac{|f(x, y)|}{x y} d x d y \\
& \leq \sum_{m=0}^{M} \sum_{n=0}^{N} \int_{\pi 2^{-m-1}}^{\pi 2^{-m}} \int_{\pi 2^{-n-1}}^{\pi 2^{-n}} \frac{1}{x y}\left(\left|\sum_{j=1}^{m} \sum_{k=1}^{n} a_{j k} \sin 2^{j} x \sin 2^{k} y\right|\right. \\
&+\left|\sum_{j=m+1}^{\infty} \sum_{k=1}^{n} a_{j k} \sin 2^{j} x \sin 2^{k} y\right| \\
&+\left|\sum_{j=1}^{m} \sum_{k=n+1}^{\infty} a_{j k} \sin 2^{j} x \sin 2^{k} y\right| \\
&\left.+\left|\sum_{j=m+1}^{\infty} \sum_{k=n+1}^{\infty} a_{j k} \sin 2^{j} x \sin 2^{k} y\right|\right) d x d y \\
&=: I_{M N}^{(1)}+I_{M N}^{(2)}+I_{M N}^{(3)}+I_{M N}^{(4)},
\end{aligned}
$$

where the empty sums equal zero by definition.

Using the trivial inequality $|\sin x| \leq|x|$, the next estimate is elementary:

$$
\begin{aligned}
I_{M N}^{(1)} & \leq \pi^{2} \sum_{m=1}^{M} \sum_{n=1}^{N} 2^{-m-n-2} \sum_{j=1}^{m} \sum_{k=1}^{n}\left|a_{j k}\right| 2^{j+k} \\
& \leq \pi^{2} \sum_{j=1}^{M} \sum_{k=1}^{N}\left|a_{j k}\right| 2^{j+k} \sum_{m=j}^{\infty} \sum_{n=k}^{\infty} 2^{-m-n-2} \\
& =\pi^{2} \sum_{j=1}^{M} \sum_{k=1}^{N}\left|a_{j k}\right| .
\end{aligned}
$$

Thus, $I_{M N}^{(1)}$ is bounded if $\sum \sum\left|a_{j k}\right|<\infty$ and this is the case if (2.2) holds.

To estimate $I_{M N}^{(4)}$ we apply an integration by substitution and then the 
Cauchy- Schwarz inequality as follows

(3.3) $I_{M N}^{(4)}$

$$
\begin{aligned}
& =\sum_{m=0}^{M} \sum_{n=0}^{N} \int_{\pi / 2}^{\pi} \int_{\pi / 2}^{\pi} \frac{1}{x y}\left|\sum_{j=m+1}^{\infty} \sum_{k=n+1}^{\infty} a_{j k} \sin 2^{j-m} x \sin 2^{k-n} y\right| d x d y \\
& \leq \sum_{m=0}^{M} \sum_{n=0}^{N}\left(\int_{\pi / 2}^{\pi} \int_{\pi / 2}^{\pi} \frac{d x d y}{x^{2} y^{2}}\right)^{1 / 2} \\
& \quad \times\left(\int_{\pi / 2}^{\pi} \int_{\pi / 2}^{\pi}\left|\sum_{j=m+1}^{\infty} \sum_{k=n+1}^{\infty} a_{j k} \sin 2^{j-m} x \sin 2^{k-n} y\right|^{2} d x d y\right)^{1 / 2} \\
& \leq \frac{1}{2} \sum_{m=0}^{M} \sum_{n=0}^{N}\left(\sum_{j=m+1}^{\infty} \sum_{k=n+1}^{\infty} a_{j k}^{2}\right)^{1 / 2} .
\end{aligned}
$$

Hence $I_{M N}^{(4)}$ is also bounded if (2.2) holds.

The estimation of $I_{M N}^{(2)}$ and $I_{M N}^{(3)}$ is an appropriate combination of the above two techniques. For example,

$$
\begin{aligned}
I_{M N}^{(2)} & \leq \pi \sum_{m=0}^{M} \sum_{n=1}^{N} \sum_{k=1}^{n} 2^{k-n-1} \int_{\pi / 2}^{\pi} \frac{1}{x}\left|\sum_{j=m+1}^{\infty} a_{j k} \sin 2^{j-m} x\right| d x \\
& \leq \pi \sum_{m=0}^{M} \sum_{n=1}^{N} \sum_{k=1}^{n} 2^{k-n-1}\left(\int_{\pi / 2}^{\pi} \frac{d x}{x^{2}}\right)^{1 / 2}\left(\int_{\pi / 2}^{\pi}\left|\sum_{j=m+1}^{\infty} a_{j k} \sin 2^{j-m} x\right|^{2} d x\right)^{1 / 2} \\
& \left.\leq \frac{\pi}{\sqrt{2}} \sum_{m=0}^{M} \sum_{n=1}^{N} \sum_{k=1}^{n} 2^{k-n-1}\left(\sum_{j=m+1}^{\infty} a_{j k}^{2}\right)^{1 / 2}\right)^{1 / 2} . \\
& =\frac{\pi}{\sqrt{2}} \sum_{m=0}^{M} \sum_{k=1}^{N}\left(\sum_{j=m+1}^{\infty} a_{j k}^{2}\right)^{1 / 2} .
\end{aligned}
$$

This is also bounded if (2.2) holds.

In an analogous way. we deduce that

$$
I_{M N}^{(3)} \leq \frac{\pi}{\sqrt{2}} \sum_{j=1}^{M} \sum_{n=0}^{N}\left(\sum_{k=n+1}^{\infty} a_{j k}^{2}\right)^{1 / 2}
$$

Combining (3.1) $-(3.5)$ yields

$$
I_{M N} \leq\left(\pi^{2}+2 \sqrt{\pi}+\frac{1}{2}\right) \sum_{m=1}^{M+1} \sum_{n=1}^{N+1}\left(\sum_{j=m}^{\infty} \sum_{k=n}^{\infty} a_{j k}^{2}\right)^{1 / 2},
$$

which completes the proof of Theorem 1 . 


\section{AuXiliary Results}

In the proof of Theorem 2 we need three lemmas.

Lemma 1. There exists a positive constant $C$ such that for any sequence $\left\{a_{j k}\right\}$ satisfying (1.3),

$$
\int_{\pi / 2}^{\pi} \int_{\pi / 2}^{\pi}\left|\sum_{j=1}^{\infty} \sum_{k=1}^{\infty} a_{j k} \sin 2^{j} x \sin 2^{k} y\right| d x d y \geq C\left(\sum_{j=1}^{\infty} \sum_{k=1}^{\infty} a_{j k}^{2}\right)^{1 / 2} .
$$

Proof. It follows from the convexity argument first applied by Orlicz [3]. (See also the proofs of [2, Lemmas 1 and 2] in the case of the two-dimensional Rademacher system. The crucial property we employ in the proof is that the double system $\left\{\sin 2^{j} x \sin 2^{l} x: j, l=1,2 \ldots ; j<l\right\}$ is orthogonal on $T_{+}$.) The integration over $[\pi / 2, \pi] \times[\pi / 2, \pi]$ instead of $T_{+}^{2}$ is justified by the substitutions $u=\pi-x$ and $v=\pi-y$.

Lemma 2. If condition (1.3) is satisfied, while condition (2.2) is not, then

$$
\sum_{j=1}^{M} \sum_{k=1}^{N}\left|a_{j k}\right|=o\left\{\sum_{m=1}^{M} \sum_{n=1}^{N}\left(\sum_{j=m}^{\infty} \sum_{k=n}^{\infty} a_{j k}^{2}\right)^{1 / 2}\right\} \quad(M, N \rightarrow \infty) .
$$

Proof. Fix a positive integer $L$, and define the integers $p_{0}$ and $q_{0}$ according to the inequalities

$$
p_{0} L<M \leq\left(p_{0}+1\right) L, \quad q_{0} L<N \leq\left(q_{0}+1\right) L .
$$

We may assume that $M, N>L$, whence $p_{0}, q_{0} \geq 1$. By the Cauchy inequality and (4.3),

$$
\begin{aligned}
\sum_{j=L+1}^{M} \sum_{k=L+1}^{N}\left|a_{j k}\right| & \leq \sum_{p=1}^{p_{0}} \sum_{q=1}^{q_{0}} \sum_{j=p L+1}^{(p+1) L} \sum_{k=q L+1}^{(q+1) L}\left|a_{j k}\right| \\
& \leq \sum_{p=1}^{p_{0}} \sum_{q=1}^{q_{0}} L\left(\sum_{j=p L+1}^{(p+1) L} \sum_{k=q L+1}^{(q+1) L} a_{j k}^{2}\right)^{1 / 2} \\
& \leq \sum_{p=1}^{p_{0}} \sum_{q=1}^{q_{0}} \frac{1}{L} \sum_{u=(p-1) L+1}^{p L} \sum_{v=(q-1) L+1}^{q L}\left(\sum_{j=u}^{\infty} \sum_{k=v}^{\infty} a_{j k}^{2}\right)^{1 / 2} \\
& \leq \frac{1}{L} \sum_{u=1}^{M} \sum_{v=1}^{N}\left(\sum_{j=u}^{\infty} \sum_{k=v}^{\infty} a_{j k}^{2}\right)^{1 / 2} .
\end{aligned}
$$


In a similar manner, we conclude that

$$
\begin{aligned}
\sum_{j=L+1}^{M} \sum_{k=1}^{L}\left|a_{j k}\right| & \leq \sum_{p=1}^{p_{0}} \sum_{k=1}^{L} \sum_{j=p L+1}^{(p+1) L}\left|a_{j k}\right| \\
& \leq \sum_{p=1}^{p_{0}} \sum_{k=1}^{L} L^{1 / 2}\left(\sum_{j=p L+1}^{(p+1) L} a_{j k}^{2}\right)^{1 / 2} \\
& \leq \sum_{p=1}^{p_{0}} \sum_{k=1}^{L} \frac{1}{L^{1 / 2}} \sum_{u=(p-1) L+1}^{p L}\left(\sum_{j=u}^{\infty} a_{j k}^{2}\right)^{1 / 2} \\
& \leq \frac{1}{L^{1 / 2}} \sum_{u=1}^{M} \sum_{k=1}^{L}\left(\sum_{j=u}^{\infty} a_{j k}^{2}\right)^{1 / 2}
\end{aligned}
$$

The symmetric counterpart of (4.5) says that

$$
\sum_{j=1}^{L} \sum_{k=L+1}^{N}\left|a_{j k}\right| \leq \frac{1}{L^{1 / 2}} \sum_{j=1}^{L} \sum_{v=1}^{N}\left(\sum_{k=v}^{\infty} a_{j k}^{2}\right)^{1 / 2} .
$$

Since $L$ is fixed and condition (2.2) is not satisfied, we infer that

$$
\sum_{j=1}^{L} \sum_{k=1}^{L}\left|a_{j k}\right|=o\left\{\sum_{u=1}^{M} \sum_{v=1}^{N}\left(\sum_{j=u}^{\infty} \sum_{k=v}^{\infty} a_{j k}^{2}\right)^{1 / 2}\right\} \quad(M, N \rightarrow \infty) .
$$

Taking into account that $L$ can be fixed as large as we want, the combination of (4.4)-(4.7) proves (4.2).

Lemma 3. If conditions (1.3), (2.4), and (2.5) are satisfied, while (2.2) is not, then

$$
\sum_{m=1}^{M} \sum_{k=1}^{N}\left(\sum_{j=m}^{\infty} a_{j k}^{2}\right)^{1 / 2}=o\left\{\sum_{m=1}^{M} \sum_{n=1}^{N}\left(\sum_{j=m}^{\infty} \sum_{k=n}^{\infty} a_{j k}^{2}\right)^{1 / 2}\right\} \quad(M, N \rightarrow \infty) .
$$

Proof. Introducing the notation

$$
A_{m k}=\left(\sum_{j=m}^{\infty} a_{j k}^{2}\right)^{1 / 2} \quad(m, k=1,2 \ldots)
$$

simplifies (4.8) as follows

$$
\sum_{m=1}^{M} \sum_{k=1}^{N} A_{m k}=o\left\{\sum_{m=1}^{M} \sum_{n=1}^{N}\left(\sum_{k=n}^{\infty} A_{m k}^{2}\right)^{1 / 2}\right\} \quad(M, N \rightarrow \infty) .
$$


Again we choose a positive integer $L$ and, analogously to (4.5), we conclude that

$$
\sum_{m=1}^{M} \sum_{k=L+1}^{N} A_{m k} \leq \frac{1}{L^{1 / 2}} \sum_{m=1}^{M} \sum_{v=1}^{N}\left(\sum_{k=v}^{\infty} A_{m k}^{2}\right)^{1 / 2} .
$$

Condition (2.4) is reformulated as

$$
\sum_{m=1}^{\infty} A_{m k}<\infty \quad(k=1,2 \ldots) .
$$

Since $L$ is fixed and condition (2.2) is not satisfied, hence it follows that

$$
\sum_{m=1}^{M} \sum_{k=1}^{L} A_{m k}=o\left\{\sum_{m=1}^{M} \sum_{n=1}^{N}\left(\sum_{k=n}^{\infty} A_{m k}^{2}\right)^{1 / 2}\right\} \quad(M, N \rightarrow \infty) .
$$

Collecting (4.10) and (4.11) yields (4.9) to be proved.

\section{Proof of Theorem 2}

We use the notation introduced in (3.1). By Lemma 1, (5.1)

$$
\begin{aligned}
I_{M N}^{(4)} & \geq \frac{4}{\pi^{2}} \sum_{m=0}^{M} \sum_{n=0}^{N} \int_{\pi / 2}^{\pi} \int_{\pi / 2}^{\pi}\left|\sum_{j=m+1}^{\infty} \sum_{k=n+1}^{\infty} a_{j k} \times \sin 2^{j-m} x \sin 2^{k-n} y\right| d x d y \\
& \geq \frac{4 C}{\pi^{2}} \sum_{m=0}^{M} \sum_{n=0}^{N}\left(\sum_{j=m+1}^{\infty} \sum_{k=n+1}^{\infty} a_{j k}^{2}\right)^{1 / 2}
\end{aligned}
$$

(cf. (3.3)). Analogously to (3.1), we also have

$$
I_{M N}^{(4)} \leq I_{M N}+I_{M N}^{(1)}+I_{M N}^{(2)}+I_{M N}^{(3)} .
$$

Now, the assumption that $(2.2)$ is not satisfied results in a contradiction. In fact, if condition (2.2) is not satisfied, then the application of Lemmas 2 and 3 (together with the symmetric counterpart of the latter) furnishes

$$
I_{M N}^{(i)}=o\left(I_{M N}^{(4)}\right) \quad(i=1,2,3) .
$$

Here we took into account estimates (3.2), (3.4), (3.5), and (5.1). By assumption $I_{M N}$ is bounded, so (5.2) contradicts (5.3). Consequently, (2.2) must be satisfied.

\section{REFERENCES}

1. R. P. Boas, Integrability theorems for trigonometric transforms, Springer-Verlag, Berlin, 1967.

2. F. Móricz, On the $|C, \alpha>1 / 2, \beta>1 / 2|$-summability of double orthogonal series, Acta Sci. Math. (Szeged) 48 (1985), 325-338. 
3. W. Orlicz, Beiträge zur Theorie der Orthogonalentwicklungen, Studia Math. 6 (1936), 2038.

4. M. C. Weiss, The law of the iterated logarithm for lacunary series and its application to the Hardy-Littlewood series, dissertation, University of Chicago, 1957.

5. A. Zygmund, Trigonometric series, Cambridge University Press, Cambridge, 1959.

Bolyai Institute, University of Szeged, Aradi Vértanúk Tere 1, 6720 Szeged, Hungary 